\title{
Eficiencia energética y monetaria de sistemas de producción de durazno (Prunus persica) en El Jarillo, Venezuela
}

\author{
Energy and monetary efficiency of peach (Prunus persica) \\ production systems in El Jarillo, Venezuela
}

Silvia Josefina Silva-Laya ${ }^{1 *}$, Humberto José Silva-Laya ${ }^{2}$ y Simón Pérez-Martínez ${ }^{3}$

\begin{abstract}
RESUMEN
La producción agrícola de durazno (Prunus persica) es la actividad comercial principal en El Jarillo (segundo productor a nivel nacional), Venezuela. En el presente trabajo se comparó la eficiencia y la productividad energética con el margen de beneficio económico de veintisiete sistemas de producción de durazno de El Jarillo. Los objetivos fueron analizar los flujos energéticos y monetarios de esos sistemas durante cuatro años $(2009,2011,2013$ y 2015) y establecer los estándares de consumo y producción para hacer producir una hectárea de durazno. El estudio fue observacional descriptivo de tipo transversal. Los sistemas fueron seleccionados con base en las subdivisiones de la localidad reconocida por los productores (Jarillo Abajo, Jarillo Centro, Enea, Tierra Caliente y La Ciénaga), la disposición de los y las productoras y los sistemas de manejo de la producción; también se consideraron las características de vegetación, suelo y clima. Los indicadores calculados y analizados fueron: eficiencia energética, productividad energética y margen de beneficio. Los sistemas resultaron ser económicamente eficientes (margen de beneficio promedio 59\%) y energéticamente deficitarios (eficiencia energética promedio de 0,58). Sustentan su productividad en el uso excesivo de insumos externos, en particular, fungicidas, gallinaza, gasolina y fertilizantes químicos (promedios entre 859.947 MJ - 604.215 MJ). En términos monetarios los costos son mayores para los rubros jornales, fertilizantes químicos y la gallinaza (promedios entre 8.195.499 Bs.F. - 1.152.395 Bs.F.). Resultó determinante el uso de la gallinaza, las fincas que la usan tuvieron menores valores en el margen de beneficio y en la eficiencia energética. En cuanto a la rentabilidad de la producción agrícola en esta zona, los elevados precios de venta del durazno permitieron obtener una utilidad promedio alrededor del $50 \%$, un valor alto a costa de una eficiencia y productividad energéticas deficitarias.
\end{abstract}

Palabras clave: eficiencia energética, productividad energética, margen de beneficio, durazno, Prunus persica.

\begin{abstract}
The agricultural production of peach (Prunus persica) is the main commercial activity in El Jarillo, Venezuela, the second biggest producer in the country. In the present work, the efficiency and the energy productivity were compared with the profit margin of twenty-seven peach's producer in El Jarillo. The objective was to analyze the energy and monetary flows of these systems over four years (2009, 2011, 2013 and 2015). The methodology used was a cross-sectional observational study for the years 2009, 2011, 2013 and 2015. The selection were based on subdivisions of the locality recognized by the producers (Jarillo Abajo, Jarillo Centro, Enea, Tierra Caliente and La Ciénaga), producers'disposal and production management systems (determined by the variety Of peach); We also considered the characteristics of vegetation, soil and climate. The indicators calculated and analyzed were: energy efficiency, energy productivity and profit margin. The systems proved to be economically efficient (average profit margin $59 \%$ ) and energy deficient (average energy efficiency 0.58). They sustain their productivity in the excessive use of external inputs, in particular fungicides, poultry, gasoline and chemical fertilizers (averages between 859,947-604,215 KJ). In monetary terms, the costs are higher for daily items, chemical fertilizers and chicken manure (averages between 8,195,499 - 1,152,395 Bs.F.). The farms that use gallinaza had lower values in the Profit Margin and Energy Efficiency. On the other hand, the high selling prices allowed obtaining an average profit around 50\%, a high value at the cost of deficit energy efficiency and productivity.
\end{abstract}

Key words: energy efficiency, energy productivity, profit margin, peach, Prunus persica.

1 Sostenibilidad, Tecnología y Humanismo, Universidad Politécnica de Cataluña. Barcelona, España.

2 Consultor independiente. Level 6203 Pacific Highway. St Leonards Nsw 20. Australia.

3 Universidad Estatal de Milagro, FACI-UNEMI. Calzada Universitaria Km. 1.5 vía Milagro-Km 26, Milagro. Guayas, Ecuador.

* Autora para la correspondencia: silvia.josefina.silva@upc.edu

Fecha de Recepción: 14 Agosto, 2017.

Fecha de Aceptación: 14 Septiembre, 2017. 


\section{Introducción}

Con la modernización agrícola, la agricultura y la ecología se separan porque los principios ecológicos se reemplazan por técnicas artificiales de control de plagas y enfermedades, fertilización y floración con productos químicos derivados de los combustibles fósiles (Iermanó y Sarandón, 2009). El distanciamiento entre agricultura y ecología incrementa el costo energético de la producción (Mäder, 2002), la pérdida de capacidad productiva de los suelos (FAO, 2015) y los impactos sociales negativos en muchas familias campesinas; además, los agroecosistemas serán insostenibles (Altieri y Toledo, 2011).

Ante esta problemática, la ciencia de la sostenibilidad contribuye con estudios de la insostenibilidad de los agroecosistemas en sus diferentes dimensiones (Funtowicz et al., 1999; Salas-Zapata et al., 2012) y presenta resultados que no son descripciones completas ni definitivas; son mediciones de aspectos que ayudan al diálogo entre los actores que intervienen en los sistemas. La agroecología aborda aspectos de la producción agrícola, como eficiencia energética y margen de beneficio económico (Lu Jianbo, 2006; Tobasura, et al., 2012) con estudios que trascienden un análisis de tipo costo-beneficio (Munda, 2004).

En escenarios de márgenes de beneficio satisfactorios pero de escasez energética es necesario un análisis que trascienda lo crematístico. A la luz de la sostenibilidad, es importante que los sistemas productivos sean económicamente favorables y socialmente aceptables, pero también deben ser energéticamente eficientes. Deben requerir menos energía para mantener la productividad en el largo plazo (Moreno et al., 2011), sin menoscabo de la salud del suelo, de la biodiversidad y de la salud humana.

En ese sentido, hay dos conceptos relacionados con el uso de la energía en los sistemas agrícolas que ayudan a analizar la productividad tomando en cuenta otros elementos: 1) la eficiencia energética, que es la relación entre las unidades energéticas producidas y las unidades energéticas invertidas y 2) la productividad energética, esto es, la relación entre la cantidad de producto obtenido y la energía invertida en el proceso productivo (Fluck y Baird, 1980; Fluck, 1995).

La importancia de realizar la investigación desde esta perspectiva radica en que en la zona de estudio es notable la ausencia de investigaciones que relacionen las dimensiones económicas y energéticas de la producción agrícola, las que guardan una relación estrecha con el manejo sostenible de los recursos naturales. Indagar sobre esta relación ofrecería información necesaria para tomar decisiones acerca de un manejo mejor de los recursos naturales.

El Jarillo ocupa el segundo lugar en producción de durazno en Venezuela con unas 500 ha distribuidas en cerca de 90 fincas productoras y comercializadoras. Las organizaciones de productores locales han dinamizado su entorno en asuntos de distribución equitativa del recurso, autogestión y satisfacción de necesidades básicas, pero esta agricultura enfrenta desafíos nuevos. Los recursos naturales sufren grave deterioro porque la tecnología productiva local y las prácticas agrícolas no tienden a conservar la vida en el suelo ni intentan disminuir la dependencia de insumos externos.

De esta zona, o del cultivo del durazno en Venezuela, no se han publicado investigaciones que tomen en cuenta el uso de la energía para la producción agrícola y que relacionen la eficiencia energética con el margen de beneficio económico. Un estudio de los flujos energéticos ofrecería información oportuna para la toma de decisiones sobre el manejo de insumos y recursos a nivel local.

Los objetivos de esta investigación fueron: 1) analizar los flujos energéticos y monetarios de estos sistemas de producción para evaluar comparativamente la eficiencia energética y monetaria y 2) establecer los estándares de consumo y producción para hacer producir una hectárea de durazno; todo esto en El Jarillo, Venezuela para los años 2009, 2011, 2013 y 2015.

\section{Materiales y Métodos}

\section{Localización del área de estudio}

La investigación se llevó a cabo en la parroquia El Jarillo, ubicada en el estado Miranda de la República Bolivariana de Venezuela. Este estado se localiza en la zona litoral central del país y ocupa una superficie de $7.950 \mathrm{~km}^{2}(0,9 \%$ del territorio nacional). Aunque desde el punto de vista de la extensión territorial es uno de los estados más pequeños de Venezuela, es el segundo en población, con aproximadamente tres millones de habitantes; por esto constituye un 
centro importante de actividades sociopolíticas, económicas, culturales y comerciales. El Jarillo es una parroquia periurbana a $34 \mathrm{~km}$ de Los Teques, capital del estado Miranda y a $75 \mathrm{~km}$ de Caracas, capital de país.

El Jarillo es un poblado establecido en una de las laderas de la cordillera de la Costa a una altitud que va desde los 1.200-2.000 msnm con temperaturas promedio anuales bastante estables de $16,7^{\circ} \mathrm{C}$. Enero y febrero son los meses más fríos $\left(15,5-16,0^{\circ} \mathrm{C}\right)$, y los más cálidos abril y mayo $\left(17,1-17,3^{\circ} \mathrm{C}\right)$. El promedio de precipitación anual es de $1.283 \mathrm{~mm}$, con meses secos de febrero y marzo y meses húmedos entre junio y noviembre.

\section{Tipo de estudio, técnicas e instrumentos}

La información recabada corresponde a los años 2009, 2011, 2013 y 2015. Para ello se diseñó un estudio observacional descriptivo transversal, dentro de la modalidad de investigación de campo. Se utilizaron fuentes vivas para la recolección de datos y se observó el evento en su contexto natural (Hernández et al., 2010).

Se usaron herramientas mixtas de recolección de datos cuantitativos. La población incluyó las 90 fincas registradas por el Ministerio de Agricultura y Tierras y se hizo un muestreo no probabilístico o dirigido. Se seleccionaron veintisiete fincas distribuidas en las cinco zonas en que los habitantes dividen el poblado (Jarillo Abajo, Jarillo Centro, Enea, Tierra Caliente y La Ciénaga). Además se tomó en cuenta la accesibilidad y disposición de los productores para participar en el estudio.

Se realizaron entrevistas estructuradas, para lo que se aplicó un instrumento previamente utilizado en esta localidad (Silva y Pérez, 2010). Este sirvió tanto de guion de entrevista como de hoja de registro de la información. Las entrevistas fueron grabadas, fotografiadas y transcritas de manera independiente para cada una de las personas encargadas de las fincas seleccionadas. El guion se diseñó con la intención de obtener datos generales sobre los productores, número de familias e integrantes, localización por GPS de las fincas, su tamaño, número de plantas de durazno, área cultivada con durazno, cosechas al año, otros rubros producidos y su destino (autoconsumo o venta).

También se indagó acerca de los insumos (químicos y biológicos) requeridos para la producción: tipo, dosis por planta, frecuencia de aplicación y costos. Así como, de los volúmenes de producción para cada año estudiado; condición de medianería (las fincas son atendidas por el núcleo familiar el que se apoya pactando con otros productores que intervienen en el proceso productivo, dividiendo los costos y beneficios a partes pactadas previamente). Esta forma de intercambio es conocida por los lugareños como medianería, producción por año y por hectárea, precio de venta y compradores. Otro punto sobre el que se indagó fue el de las labores realizadas para los cultivos y las horas hombre destinadas a estas.

Los indicadores del estudio fueron dos de funcionamiento energético para agroecosistemas de producción de alimentos y uno financiero (Chamorro et al., 2015) (Tabla 1). La literatura reporta que los indicadores de eficiencia energética son una herramienta adecuada para el análisis integral de los agroecosistemas (Abbona et al. 2007). Se hizo una adaptación respecto a la referencia original: solo se consideró el área de cada finca dedicada a la producción de durazno, cultivo principal y emblemático de la localidad. Todos los cálculos están realizados con base en una hectárea de durazno.

\section{Para el análisis de los resultados}

Se delimitó el área a las hectáreas dedicadas a la producción del durazno como unidad básica para el análisis de los flujos energéticos y monetarios (entrada/salida). Algunos autores hacen una distinción entre gastos de energía directos e indirtectos (Baird et al., 1997). En este estudio no se hizo tal distinción; todos los gastos se consideraron como energía insumida. Los fungicidas, herbicidas, desfoliantes y plaguicidas se relacionaron en forma lineal con la dosis aplicada. Para los fertilizantes químicos se consideró la concentración del nutriente en el producto comercial contenida en la dosis aplicada. Todos los cálculos energéticos se conviertieron en unidades equivalentes a Megajoules (Tabla 2).

La energía producida (salida) se calculó multiplicando las unidades de energía equivalentes al durazno por el total de la producción. La energía insumida (entrada) se calculó multiplicando el equivalente energético de cada insumo por el total de la cantidad consumida. Luego se relacionó la producción con cada unidad de energía que se invirtió, el coeficiente de esta relación salida/ 
Tabla 1. Indicadores energéticos y financieros empleados en el estudio.

\begin{tabular}{|c|c|c|c|}
\hline Tipo & Indicador & Descripción & Fórmula \\
\hline \multirow{4}{*}{ 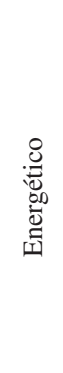 } & & $\begin{array}{l}\text { EP: Cantidad de energía producida, expresado en Megajoules por } \\
\text { año }(\mathrm{MJ} / \mathrm{año}) . \mathrm{EP}=\text { (producción * energía })\end{array}$ & \multirow{3}{*}{$\mathrm{EE}=\mathrm{EP} / \mathrm{EI}$} \\
\hline & Eficiencia energética (EE) & $\begin{array}{l}\text { EI: Equivalente energético de la cantidad del insumo gastado en la } \\
\text { producción (en MJ) por año. EI = (Gasto*Energía) }\end{array}$ & \\
\hline & & $\begin{array}{l}\text { Gasto }=\left(\mathrm{N}^{\circ} \text { de plantas }\right) *(\text { Dosis por planta }) *(\text { Cosechas al año }) * \\
(\text { Aplicac/cosecha })\end{array}$ & \\
\hline & Productividad energética (PE) & $\begin{array}{l}\text { Relación entre la cantidad de producto obtenido }(\mathrm{Pr}=\text { producción }) \text { y } \\
\text { la energía insumida }(\mathrm{EI}) \text { en el proceso de producción }\end{array}$ & $\mathrm{PE}=\mathrm{Pr} / \mathrm{EI}$ \\
\hline \multirow{2}{*}{ 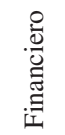 } & \multirow{2}{*}{ Margen de beneficio (MB) } & Ingresos totales: IT = (PVP) * (Producción) & \multirow{2}{*}{$\mathrm{MB}=\mathrm{IT}-\mathrm{CT} / \mathrm{IT}$} \\
\hline & & Costos totales: $\mathrm{CT}=$ Cantidad insumo $*$ Costo unitario & \\
\hline
\end{tabular}

Tabla 2. Equivalentes energéticos según varios autores.

\begin{tabular}{|c|c|}
\hline Producto & $\begin{array}{c}\text { Contenido } \\
\text { energético } \\
\text { (mj /unidad) }\end{array}$ \\
\hline \multicolumn{2}{|l|}{ Insumos (Zentner, 2004) } \\
\hline Defoliante (kg) & 238 \\
\hline Fósforo (kg) & 5 \\
\hline Nitrógeno (kg) & 57 \\
\hline Urea $(\mathrm{kg})$ & 57 \\
\hline Potasio (kg) & 7 \\
\hline Gallinaza (kg) & 11 \\
\hline Fungicida (kg) & 104 \\
\hline Herbicida (kg) & 238 \\
\hline Insecticida (kg) & 184 \\
\hline Gasolina (L) & 39 \\
\hline \multicolumn{2}{|l|}{ Herramientas (Pimentel, 1991) } \\
\hline $\begin{array}{l}\text { Unidades de sistema de riego, fertilizadoras, } \\
\text { fumigadoras, mangueras alta presión, } \\
\text { Asperjadores }\end{array}$ & 88 \\
\hline \multicolumn{2}{|l|}{ Mano de obra (Zentner, 2004) } \\
\hline Mano de obra (horas hombre) & 1,05 \\
\hline \multicolumn{2}{|l|}{ Otros (Zentner, 2004) } \\
\hline Electricidad (KW) & 3,60 \\
\hline Durazno $(\mathrm{kg})$ & 1,63 \\
\hline
\end{tabular}

entrada es la unidad de energía que produce el sistema por cada unidad de energía invertida, es decir, la eficiencia energética (Pimentel, 1991).

En cuanto a los flujos monetarios, para calcular el margen de beneficio económico se consideraron las entradas: multiplicando la producción anual de durazno, en $\mathrm{kg}$, por el precio de venta en el mercado para cada año y las salidas: multiplicando los costos de mercado de cada año por las cantidades de insumo gastado en el proceso productivo. Como costos fijos para poner a funcionar el sistema se consideró: la electricidad y la depreciación de las herramientas. Todos los costos de los insumos y los precios de la producción fueron los valores de mercado para cada período estudiado. Finalmente, se calculó el margen de beneficio (Tabla 1).

\section{Resultados y Discusión}

\section{Eficiencia energética y productividad energética en el cultivo de durazno de El Jarillo}

El análisis de los flujos energéticos de la producción de durazno en los sistemas estudiados mostró que el mayor peso de las entradas energéticas recae sobre la gallinaza y los fungicidas, los que representan el 50\% del total de energía insumida. Un comportamiento similar se mantuvo en los cuatro períodos (Tabla 3). Comparando los tipos de insumo entre los sistemas se encontró que casi el 100\% de la energía que el sistema necesita para producir el durazno es energía externa a la finca. La mano de obra representa menos de $1 \%$.

Este alto porcentaje de insumos hace que la eficiencia energética del sistema resulte por debajo de la unidad; por ejemplo, para los años 2009, 2011 y 2013 el promedio de la eficiencia 
Tabla 3. Flujos energéticos en los sistemas de cultivo de durazno, de El Jarillo, Venezuela, para el período 2009/2015 (expresado en megajoules).

\begin{tabular}{lccrrr}
\hline \multicolumn{1}{c}{ Categoría de la energía } & 2009 & 2011 & 2013 & 2015 & Promedios \\
\hline Fungicida & \multicolumn{5}{c}{ Entradas } \\
Gallinaza & 949.018 & 927.153 & 893.495 & 670.121 & 859.947 \\
Gasolina & 952.317 & 870.480 & 788.642 & 591.481 & 800.730 \\
Fertilizantes químicos & 675.675 & 657.638 & 634.725 & 476.044 & 611.020 \\
Defoliante & 663.965 & 649.158 & 630.707 & 473.031 & 604.215 \\
Electricidad & 328.232 & 317.921 & 305.290 & 228.968 & 295.103 \\
Herramientas & 133.650 & 130.082 & 125.550 & 94.163 & 120.861 \\
Herbicida & 65.331 & 63.704 & 61.461 & 46.096 & 59.148 \\
Mano de obra & 38.456 & 35.563 & 32.669 & 24.502 & 32.798 \\
Insecticida & 13.052 & 12.703 & 2.261 & 9.195 & 11.803 \\
Total & 9.733 & 9.733 & 9.733 & 7.300 & 9.125 \\
& 3.829 .428 & 3.674 .135 & 3.494 .534 & 2.620 .900 & 3.404 .749 \\
\hline Total producción de durazno & 2.096 .100 & 1.995 .569 & 1.924 .169 & 1.924 .169 & 1.985 .002 \\
Eficiencia energética & $\mathbf{0 , 5 5}$ & $\mathbf{0 , 5 4}$ & $\mathbf{0 , 5 5}$ & $\mathbf{0 , 7 3}$ & $\mathbf{0 , 5 8}$ \\
\hline
\end{tabular}

energética resultó de 0,55 , lo que significa que con una unidad de energía que ingresa al sistema este solo es capaz reproducir 0,55. Esta situación hace que los sistemas sean deficitarios en términos de energía. En el estudio se observó que para el año 2015 hubo una mejora en la eficiencia energética la que pasó de 0,55 a 0,73 ; sin embargo, aun con la mejora el sistema sigue siendo deficitario. Esta mejora, que se puede observar en la Tabla 3, obedece, básicamente, a que por la escasez de productos químicos que ha vivido el mercado venezolano producto de la crisis política y socioeconómica, los productores y productoras se han visto obligados a disminuir la aplicación de productos químicos.
Debido a que se encontraron sistemas que producen una y media cosecha al año y sistemas con dos cosechas por año, la salida de energía por año varía para los diferentes sistemas, aunque tengan igual número de plantas o la misma cantidad de hectáreas cultivadas. No obstante, esta variación en las cosechas al año no repercute en la mejora de la eficiencia energética.

Referente a la productividad energética, se estableció la relación entre la unidad del producto por cada MJ (energía invertida) utilizado en el proceso de producción y se obtuvo que la mayor productividad energética la tiene la finca 11 , con un valor de 0,81 kilogramos de durazno por MJ invertido, para el año 2015. La finca 13 resultó

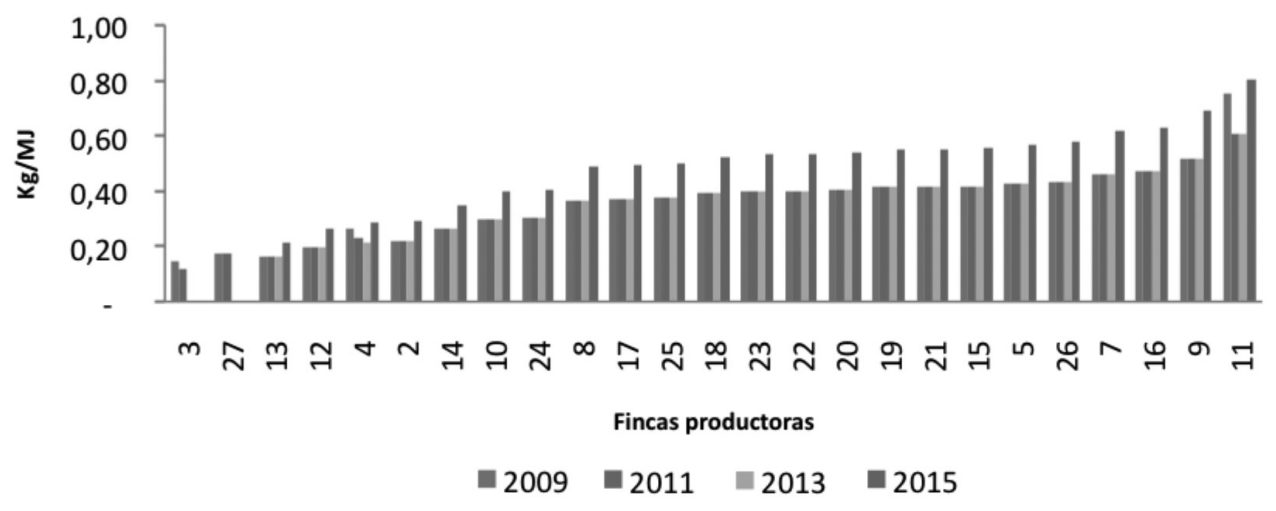

Figura 1. Productividad energética de los sistemas de producción de durazno de El Jarillo (período 2009-2015). 
con la menor productividad energética de $0,22 \mathrm{~kg}$ de durazno por MJ. La relación de productividad entre las dos fincas es 1:0,27; es decir, la finca 11 produce 0,27 veces más durazno que la finca 13 por cada unidad energética (MJ) utilizada; lo que indica que no hay una diferencia sustancial en la productividad energética a pesar de que la finca 11 duplica el uso de productos químicos, tanto para provocar la floración como para fertilizar el suelo.

\section{Beneficios económicos en el cultivo de durazno de EI Jarillo}

El mayor costo económico recae sobre la mano de obra, los fertilizantes químicos y la materia orgánica (Tabla 4). La mano de obra se lleva casi el $50 \%$ del costo. El siguiente porcentaje lo ocupan los fertilizantes (químicos y gallinaza) pero el precio de estos insumos externos, comparado con el precio de venta del durazno, no impide que el durazno sea un producto económicamente rentable para los productores. En esta Tabla 4 se observa, también, un incremento considerable en los flujos energéticos desde el año 2009 hasta el 2015, este incremento obedece, básicamente, a la inflación del país para cada período estudiado.

El estudio muestra que el $42 \%$ de los costos monetarios y energéticos recae sobre los fertilizantes, ambos externos a la finca. El margen de beneficio relaciona los valores monetarios de los ingresos y los gastos en los que se incurre durante un año de actividad económica. Al utilizar el mismo período para las veintisiete fincas, el indicador permite comparar la eficiencia monetaria de la producción de durazno de las fincas estudiadas (datos no mostrados).

El mejor desempeño, desde el punto de vista costo-beneficio, considerando los precios de venta y costos del mercado en cada período, 2009, 2011, 2013 y 2015, lo mostraron las fincas con los códigos: $8,10,11,25$ y 25 , con valores entre $66 \%$ y $41 \%$ (según análisis de conglomerados de este indicador, correlación cofenética 0,87 , datos no mostrados). Dieciséis fincas, la mayoría, tuvieron un margen de beneficio entre $27 \%$ y $44 \%$, y las de menores valores mostraron resultados entre $5 \%$ y $16 \%$. Para las las fincas 3 y 27 solo se promediaron los años 2009 y 2011 porque cambiaron de actividad productiva durante los siguientes períodos del estudio. Las diferencias radican principalmente en el uso de la gallinaza como fuente de fertilización, las fincas que usan este fertilizante fueron las que menor margen de beneficio y menor eficiencia energética mostraron.

Los elevados precios de venta del durazno, durante este período estudiado, les permitió obtener una utilidad promedio que se mantuvo alrededor del $50 \%$, un margen de beneficio alto a costa de

Tabla 4. Flujos monetarios* de los sistemas de cultivo de durazno, de El Jarillo, Venezuela, para el período 2009/2015 (expresado en bolívares).

\begin{tabular}{lrrrrr}
\hline \multicolumn{1}{c}{ Categoría Monetaria } & \multicolumn{1}{c}{2009} & \multicolumn{1}{c}{2011} & \multicolumn{1}{c}{2015} & Promedio \\
\hline \multicolumn{5}{c}{ Salidas } \\
\hline Mano de obra & 1.418 .268 & 2.445 .161 & 4.141 .398 & 23.776 .959 & 8.195 .499 \\
Fertilizantes químicos & 1.165 .890 & 1.479 .943 & 3.262 .685 & 4.894 .028 & 2.700 .636 \\
Gallinaza & 634.878 & 754.416 & 1.288 .115 & 1.932 .173 & 1.152 .395 \\
Fungicida & 172.982 & 222.108 & 917.545 & 1.376 .310 & 672.236 \\
Defoliante & 12.470 & 20.025 & 368.228 & 552.341 & 238.266 \\
Herramientas & 45.559 & 57.813 & 71.132 & 106.534 & 70.259 \\
Gasolina & 17.325 & 21.921 & 39.874 & 59.811 & 34.733 \\
Electricidad & 37.125 & 36.134 & 34.875 & 26.156 & 33.573 \\
Herbicida & 2.878 & 3.497 & 9.340 & 14.009 & 7.431 \\
Insecticida & 2.283 & 2.969 & 5.595 & 8.392 & 4.810 \\
Total & 3.509 .659 & 5.043 .985 & 10.138 .786 & 32.746 .712 & 13.109 .838 \\
\hline & \multicolumn{7}{c}{ Entradas } & & \\
\hline Venta de durazno & 5.351 .563 & 8.151 .833 & 15.327 .325 & 29.475 .625 & 23.517 .526 \\
Total & 5.351 .563 & 8.151 .833 & 15.327 .325 & 29.475 .625 & 23.517 .526 \\
Margen de beneficio & $52 \%$ & $62 \%$ & $51 \%$ & $63 \%$ & $59 \%$ \\
\hline
\end{tabular}

* El incremento considerable de los flujos monetarios obedece a la inflación. 
una eficiencia y productividad energética que está por debajo de la unidad (Figura 2). Es decir, hay una producción promedio de energía al año de 1.985.002 MJ y un promedio de energía ingresada, en forma de insumos externos, de 3.404.709 $\mathrm{MJ}$ al año, por lo tanto la relación salidas/entradas resulta en 1:0.58, lo que significa que por cada unidad de energía invertida solo se obtiene 0.58. Esto muestra al sistema como energéticamente ineficiente; el 99\% de la energía que utiliza el sistema se concentra en los insumos externos a la finca (Tabla 3 ).
Esta forma de manejo, basada en el uso de insumos externos, para enfrentar problemas de fertilización del suelo, manejo de plagas y enfermedades y para provocar artificialmente la floración con fines de obtener más de una cosecha por año desfavorece la autosuficiencia y, por lo tanto, atenta contra la sostenibilidad de los sistemas productivos en el largo plazo (Blandi et al., 2013), debido al deterioro del capital natural.

Las fincas que resultaron con mayores y menores resultados, se muestra en la Tabla 5.

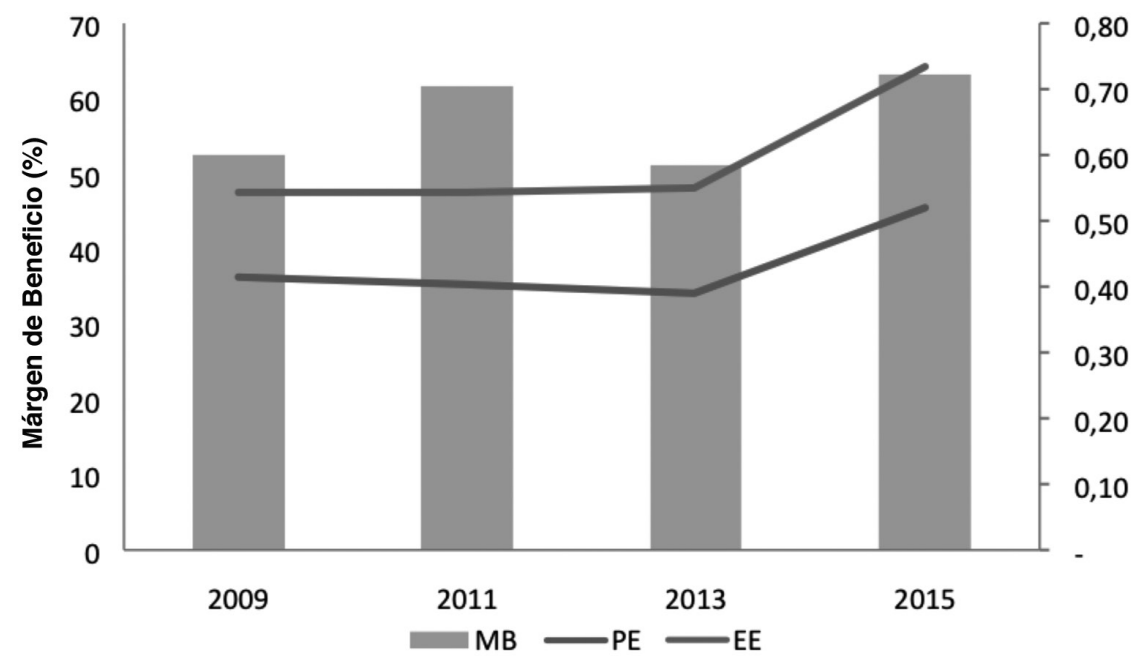

Figura 2. Resultado de los indicadores eficiencia energética (EE), productividad energética (PE) y margen de beneficio (MB de los sistemas de producción de durazno de El Jarillo (período 2009-2015).

Tabla 5. Características de las fincas con mayor y menor eficiencia energética.

\begin{tabular}{lrr}
\hline \multicolumn{1}{c}{ Indicadores } & $\begin{array}{r}\text { Mayor eficiencia }(0,82) \\
\text { Finca } 11\end{array}$ & $\begin{array}{c}\text { Menor eficiencia }(0,35) \\
\text { Finca } 4\end{array}$ \\
\hline Hectáreas cultivadas & 3,5 & 5 \\
Número de plantas & 230 & 500 \\
Densidad de plantación (plantas/ha) & 65 & 100 \\
Cosechas al año & 2 & 1,5 \\
Uso de insumos (por planta): & & \\
Defoliantes (kg) & 0,09 & 0,19 \\
Fertilizantes químicos (kg) & 1,63 & 11 \\
Gallinaza (kg) & 0 & 107 \\
Fungicida (kg) & 0,40 & 0,23 \\
Herbicida (kg) & 0,14 & 0 \\
Insecticida (kg) & 0 & 0,06 \\
Promedio de indicadores: & & \\
Producción (kg/ha/año) & 48.000 & 90.000 \\
Productividad energética & 1,48 & 0,91 \\
Eficiencia energética & 0,82 & 0,35 \\
Margen de beneficio & $66 \%$ & $15 \%$ \\
\hline
\end{tabular}


Las diferencias más significativas se sustentan en la cantidad de insumos externos a la finca utilizados para hacer producir cada sistema, más específicamente en la utilización de fertilizantes químicos y fungicidas (Tabla 5). La finca con mejores resultados no usa gallinaza y usa tres veces menos cantidad de fertilizantes químicos. Esta diferencia en el uso del insumo le representa a la finca 11 una mejora energética de más del $100 \%$, con respecto a la finca 4 .

\section{Estándares de consumo y producción}

Según el período analizado y los datos a los que se tuvo acceso, una hectárea de durazno con 100 plantas puede producir $30.000 \mathrm{~kg}$ año ${ }^{-1}$, según se detalla en la Tabla 6. Estos valores sirven de referente en condiciones donde la inflación y las fluctuaciones de precios son significativas.

La mayoría de los sistemas agrícolas modernos que dependen de los combustibles fósiles son ineficientes desde el punto de vista energético $\mathrm{y}$, a largo plazo, insostenibles. Las prácticas agrícolas sanas deben priorizar el uso de energías renovables, para conservar la energía fósil y los recursos de suelo, agua y biológicos (Pimentel y Pimentel, 2005). Estos sistemas estudiados son un ejemplo de ello, cada unidad de energía usada para producir, en el mejor de los resultados, solo devuelve el $73 \%$ de lo invertido, es decir, el sistema tiene un déficit energético de $37 \%$.

Evaluar sistemas agroproductivos con atención en los flujos energéticos y monetarios de los sistemas permite entender cómo se sustenta la productividad, desde el punto de vista de la energía necesaria para sacar adelante la producción (Gliessman, 2002). Estos sistemas de El Jarillo han alcanzado una producción económicamente eficiente a base de implementar una tecnología local dependiente de productos químicos; descartan manejos basados en los procesos naturales que les permitirían tener organismos con funciones ecológicas fundamentales que podrían optimizar los procesos del ecosistema y hacer que este se autorregule. Por ejemplo, se podría mejorar la eficiencia en el uso de los recursos naturales locales, como las plantas fijadoras de nitrógeno (leguminosas) (Paredes, 2013) o las que actúan como repelentes naturales (Vázquez et al., 2008; Elkinton, 2007), o el manejo de la biodiversidad para el control biológico de plagas (Nicholls, 2006).

El estudio mostró que el $42 \%$ de los costos monetarios y energéticos recae sobre los fertilizantes externos a la finca; si hubiera una tendencia a mejorar la fertilización del suelo de manera natural, aprovechando mejor los desechos orgánicos e incorporando prácticas sanas de conservación de la vida en el suelo esto repercutiría en un mejor uso de la energía y a la larga volvería a estos sistemas más sostenibles.

\section{Conclusiones}

La tecnología productiva local y las prácticas agrícolas de las fincas estudiadas atentan contra la sostenibilidad de la producción en el largo plazo. Las fincas sustentan su productividad en el auxilio de productos químicos externos. Utilizan defoliantes para provocar la floración y obtener más de una cosecha por año. Los problemas de

Tabla 6. Estándares de consumo y producción para 100 plantas de durazno en un año.

\begin{tabular}{lclr}
\hline \multicolumn{1}{c}{ Descripción } & Cantidad & Descripción & Cantidad \\
\hline \multicolumn{4}{c}{ Entradas } \\
\hline \multicolumn{4}{c}{ Insumos (kg) } \\
Defoliante & 60 & Costos Fijos \\
Fungicida & 369 & Electricidad (Kw) & 1.473 \\
Insecticida & 18 & Jornal (horas) & 714 \\
Herbicida & 33 & & 660 \\
Fertilizantes químicos & 2.588 & \\
Gallinaza & 2.625 & \\
\hline \multicolumn{4}{c}{ Salidas } \\
\hline Producción durazno & 30.000 & \\
\hline
\end{tabular}


plagas y enfermedades los enfrentan con el uso de insecticidas y fungicidas y para lograr la fertilización del suelo recurren a fertilizantes químicos y gallinaza. El alto uso de insumos externos repercute en un elevado costo energético que vuelve a los sistemas energéticamente deficitarios. Sin embargo, a la luz de los indicadores calculados y analizados, las fincas son económicamente rentables porque el precio de venta del durazno cubre suficientemente los costos monetarios. Los sistemas son altamente dependientes de insumos externos, de estos los que más inciden en el aumento de los costos, tanto energéticos como monetarios, son los fungicidas y el fertilizante gallinaza.

\section{Agradecimientos}

Agradecemos la colaboración esmerada de Lucas Gerik y Florencia Ardían y a sus familias por su empeño en participar en el estudio y en ayudarnos con los demás productores y productoras para que nos recibieran en sus fincas y estuvieran abiertas a responder nuestras preguntas.

\section{Literatura Citada}

Abbona, E.; Blandi, S. et al., 2013 y Marasas, M.

2007. Análisis de la eficiencia energética en viñedos tradicionales de Berisso, Argentina. Revista Brasileira Agroecología, 2: 1449-52.

Altieri, M. Y Toledo, V.

2011. The agroecological revolution of Latin America: rescuing nature, securing food sovereignity and empowering peasants. J. Peasant Studies, 38: 587-612.

Baird, G.; Acorn, A. y Haslam, P.

1997. The energy embodied in building mateirals-update New Zeland coefficients and their significance. IPENZ Transaction, 24: 46-54.

Blandi, M.; Paleologos, M.; Sarandón, S. y Veiga, I.

2013. Identificación de impedimentos para avanzar hacia una conducta sustentable en pequeños horticultores de La Plata, Argentina. Cuadernos Agroecología, 8: 1-5.

Elkinton, J.

2007. El papel de la ecología de poblaciones y de los modelos de población en el control biológico. En: Van Driesche, R.; Hoddle, M.; Reardon, R. Control de plagas ymalezas por enemigos natuFAO rales. FHTET. Washington, D.C. US. pp. 153-178.

2015. Energy-smart food for people and climate. Issue paper. Food and Agriculture Organization of the United Nations Rome, Italy. 68 p.

Fluck, R.

1995. The hidden input. Southern Regional Workshop Evaluating Sustainability. Florida, USA. University of Florida. pp. 31-43.

Fluck, R.C. and Baird, C.D.

1980. Agricultural Energetic. Avi Publishing Company, Inc. Gainesville University of Florida. US. 192 p.

Funtowicz, S.; Martínez-Alier, J. y Ravetz, J. 1999. Information tools for environment policy under conditions of complexity. Environmental Issues Series 9. European Environmental Agency. Copenhagen, Denmark. 34 p.

Gliessman, $\mathrm{S}$.

2002. Agroecología. Procesos ecológicos en agricultura sustentable. CATIE. San José, Costa Rica. 359 p.

Gliessman, S.

2013. Agroecología: Plantando raíces de la resistencia. Agroecología, 8 (2): 19-26.

Iermanó, M. y Sarandón, S

2009. ¿Es sustentable la producción de agrocombustibles a gran escala? El caso del biodiesel en Argentina. Revista Brasileira de Agroecología, 4 (1): 4-17.
Lu Jianbo

2006. Energy balance and economic benefits of two agroforestry systems in northern and southern China. Agriculture, Ecosystems \& Environment, 116: 255-262.

Mäder, $\mathrm{P}$

2002. Soil Fertility and Biodiversity in Organic Farming. Science, 3 (5): 94-97.

Moreno, M.; Lacasta, C.; Meco, R. y Moreno, C.

2011. Rainfed crop energy balance of different farming systems and crop rotations in a semi-arid environment: Results of a longterm trial. Soil \& Tillage Research, 114 (1): 18-27.

Munda, G.

2004. Métodos y procesos multicriterio para la evaluación social de las políticas públicas. Revista Iberoamericana de Economía Ecológica, 1 (19): 31-45.

Nicholls, C.

2006. Bases Agroecológicas para Diseñar e Implementar una Estrategia de Manejo de Hábitat para Control Biológico de Plagas. Agroecología, 1 (2006): 37-48.

Paredes, M.

2013. Fijación biológica de nitrógeno en leguminosas y gramíneas. Agroecología, 9 (2): 9-18.

Pimentel, D.; Berardi, G. y Fast, S.

1991. Energy efficiencies of farming wheat, corn, and potatoes organically. In: Organic Farming Current Technology, its Role in Sustainable Agriculture, ASA, USA. Special publication, 46: 151-161.

Pimentel, D. y Pimentel, M.,

2005. Energy use in agriculture: an overview. Mag. Low External Input Sustain. Agric. 21 (1): 5-7.

Salas-Zapata, W.; Ríos-Osorio, L. y Álvarez-Del Castillo, J.

2012. Marco conceptual para entender la sustentabilidad de los sistemas socioecológicos. Ecología Austral, 22: 74-79.

Silva, S. y Pérez, S.

2010. Sustentabilidad de fincas productoras de durazno en El Jarillo, estado Miranda, Venezuela. RET. Revista de Estudios Transdisciplinarios, 2: 45-62.

Silva, S. y Pérez, S.

2016. Evaluación agroecológica de sistemas hortícolas de dos zonas del oriente antioqueño, Colombia. Revista Colombiana de Ciencias Hortícolas, 10 (2): 355-366.

Tobasura, I.; Moreno, F.; Aya, S. y Mora, J.

2012. Productividad energética y monetaria en fincas campesinas del departamento de Caldas. Tres estudios de caso. Luna Azul, 34: 101-112. 
Vázquez, L. y Martínez, H.

2015. Propuesta metodológica para la evaluación del proceso de reconversión agroecológica. Agroecología, 10 (1): 33-47.
Zentner, R.; Lafond, G.; Derksen, D.; Nagy, C.; Wall D. y May, W. 2004. Effect of tillage method and crop rotation on non renewable energy use efficiency for a thin Black Chernozem in the Canadian Prairies. Soil and Tillage Research, 77: 125-136. 